\title{
A permutation invariant collective variable to track and drive vacancy dynamics in simulations of solids
}

\author{
Jan M. Knaup.* Michael Wehlau, and Thomas Frauenheim \\ Bremen Center for Computational Materials Science, \\ University of Bremen, Am Fallturm 1, D-28359 Bremen, Germany
}

\begin{abstract}
Vacancy dynamics in oxides are vital for understanding redox reactions and resulting memristive effects or catalytic activity. We present a method to track and drive vacancies which we apply to metadynamics simulation of oxygen vacancies $\left(\mathrm{V}_{\mathrm{O}}^{2+}\right)$ in rutile, demonstrating its effectiveness. Using the density functional based tight binding method, it is possible to explore the free energy hyperplane of oxygen vacancies in $\mathrm{TiO}_{2}$. We show that the migration of $\mathrm{V}_{\mathrm{O}}^{2+}$ in $\mathrm{TiO}_{2}$ is governed by the jump with the highest degree of topological interconnection. Free energy profiles are consistent with minimum energy paths.
\end{abstract}

PACS numbers: $\quad 61.72 . j d, 66.30 . \mathrm{Lw}, 02.70 . \mathrm{Ns}$

Transition metal oxides exhibit a highly complex behavior, with strong coupling between electronic and ionic transport processes, making them highly interesting as electronic materials beyond their traditional use as dielectrics. The most prominent example of such effects is the memristive behavior ${ }^{1}$ of $\mathrm{TiO}_{2-x}$ and many other substoichiometric oxides 2 . In titania, the memristive behavior is linked to the formation and dissolution of conducing filaments ${ }^{1}$ or layers ${ }^{3}$ of room-temperature metallic $\mathrm{Ti}_{n} \mathrm{O}_{2 n-1}$ Magnéli phases ${ }^{4}$ in the insulating $\mathrm{TiO}_{2}$. These phases of titania are modifications of the rutile crystal structure in which oxygen vacancies $\left(\mathrm{V}_{\mathrm{O}}\right)$ are ordered in slip-planes. In order to understand their formation and dissolution it is essential to understand the dynamics of oxygen vacancies aggregating and dispersing.

Yet, the memristive effect is far from being the only interesting physics of metal oxides in which vacancy dynamics play a crucial role. Catalytic effects at oxide surfaces can rely on the presence and ability to replenish surface vacancies ${ }^{56}$ or $\mathrm{O}$ ion transport through the catalyst 5 . To be efficient, the latter relies on vacancies and can in fact be mapped to a vacancy transport process in the reverse direction. Oxide based (photo)catalysis is regarded as one of the most promising fields of renewable energy conversion.

These examples show that complex physical processes at the surface and in the bulk of reducible metal oxides require intimate understanding of the dynamical behavior of $\mathrm{V}_{\mathrm{O}}$ defects and the relevant driving forces. Many of these processes are rare events, i.e. their activation energy is larger than the average thermal energy at relevant conditions, which makes them inaccessible to unbiased molecular dynamics simulation. Techniques for rare event simulation ${ }^{811}$ rely on reaction coordinates describing the transition of interest. In most cases, the reaction coordinate is required to be continuous and at least piecewise differentiable with respect to the atomic positions $r_{i}^{\overrightarrow{\text { lat }}}$. Achieving this for a vacancy coordinate $\overrightarrow{r_{\mathrm{v}}}$ is difficult, as vacancies are not directly simulated objects but an emergent property. In the strictest sense, the position of a vacancy cannot be continuous, as it is defined as an unoccupied lattice position. Yet, since a vacancy can change its position, albeit indirectly, a generalization of the vacancy position to allow the description of the transition is possible. So far, studies of vacancy migration focus on single hopping events, tracing the position of the moving neighbor atom, and implicitly defining the vacancy motion as the opposite of the ion motion 12 15. This auxiliary definition suffers from the conceptual problem, that at or at least close to the transition state the vacancy defined as "where no ion is" coincides with the position of the moving ion. Of greater practical impact is that focusing on a single ion when driving the vacancy severely limits the search space for the vacancy transition path. The a-priori assumption that the motion of only the selected atom alone leads to the vacancy motion precludes any collective effects.

One approach to capture the emergent nature of vacancies is to analyze the simulated volume for voids and designate such spaces as vacancies based on their size (e.g.16[17). While highly flexible and useful to detect an unknown number of vacancies in a matrix of unspecified crystal structure, the approach is computationally cumbersome and can neither yield a continuous $\partial \overrightarrow{r_{\mathrm{v}}} / \partial r_{i}^{\overrightarrow{\text { lat }}}$ at acceptable computational effort, nor can it describe the crucial hopping events.

We return our focus to the discrete reference positions. In the transition state, the moving ion is equally far from two lattice positions ${ }^{18}$, which suggests having two vacancies while only one ion is missing. This leads us to the desired generalization of the vacancy concept allowing for the continuous tracking of vacancy motion: Instead of a Boolean assignment of ions to lattice positions, ions may impart a certain amount of occupiedness to lattice sites, ranging from $0 \ldots 1$ depending on their distance. In this picture the ion at the transition state partially occupies both adjacent lattice positions. $\overrightarrow{r_{\mathrm{v}}}$ is then defined as the average of the lattice positions weighted by unity minus their occupiedness. In contrast to coordinates derived from specific ion positions, this concept is inherently invariant under permutation of the ionic labels, removing many a-priori constraints. We therefore call this coor- 
dinate Permutation Invariant Vacancy Location Trajectory, in short PIVoT. Mathematically, the position of the vacancy is determined from the externally supplied reference lattice positions $\overrightarrow{r_{i}^{\text {lat }}}$ and the ionic positions $\overrightarrow{r_{j}^{\text {ion }}}$ by a weighted average

$$
\begin{aligned}
\overrightarrow{r_{\mathrm{v}}} & =\sum_{i} r_{i}^{\overrightarrow{\mathrm{lat}}} \frac{w_{i}}{\sum_{i} w_{i}}\left[\frac{G_{i}}{\sum_{i} G_{i}}\right] \\
w_{i} & =1-\frac{1}{\exp \left(\left(r_{\min , i}-d_{0}\right) / \tau\right)+1} \\
G_{i} & =\exp \left(\frac{-\left|r_{i}^{\overrightarrow{\mathrm{lat}}}-\overrightarrow{r_{\mathrm{v}}}\right|^{2}}{2\left(\sigma_{\mathrm{G}}\right)^{2}}\right) \\
r_{\min , i} & =\min _{j}\left(\left|r_{i}^{\text {lat }}-r_{j}^{\overrightarrow{\text { ion }}}\right|\right)
\end{aligned}
$$

where the weighting function $w_{i}$ is a mirrored FermiDirac distribution function with the switchover distance $d_{0}$ and a steepness parameter $\tau$. It was chosen for being able to control the smooth transition with convenient parameters. For tracing a vacancy in the post-processing of existing trajectories, $d_{0}$ and $\tau$ are chosen such that the weight of a lattice position remains almost zero as long as the closest ion does not stray outside the range of thermal oscillation. For use as a collective variable (CV) in meta dynamics, the derivative of the $\mathrm{CV}$ with respect to atomic coordinates would be zero close to equilibrium and the algorithm could not drive the dynamics. In this case, $d_{0}$ and $\tau$ are chosen to yield a smoother transition and weight thermal displacements. Since the global averaging of all $\overrightarrow{r_{i}^{\mathrm{lat}}}$ will now draw $\overrightarrow{r_{\mathrm{v}}}$ towards the center of gravity of the $r_{i}^{\overrightarrow{\mathrm{lat}}}$, the term $G_{i}$ needs to be applied to additionally weight the $r_{i}^{\overrightarrow{\text { lat }}}$ by their distance from $\overrightarrow{r_{\mathrm{v}}}$. In this case Eq. 1 has to be solved self consistently, with the $\sigma$ parameter chosen so that the FWHM of $G_{i}$ falls between the 2 nd and 3rd shells of the moving species.

In our simulations of $\mathrm{V}_{\mathrm{O}}^{2+}$ diffusion in $\mathrm{TiO}_{2}$ we calculate energies and forces using the self-consistent charge version of the density-functional based tight-binding $\operatorname{method}^{19}$ (SCC-DFTB) with the tiorg parametrization 20. We iterate SCC until charge errors are $<10^{-8} \mathrm{e}^{-}$. Supercells between $2 \times 2 \times 3$ and $4 \times 4 \times 6$ rutile units, each containing a single $\mathrm{V}^{2+} \mathrm{O}$ defect were simulated, using a 2x2x2 Monkhorst-Pack $k$-point set 21 . The $2+$ charge state was chosen based on the results in ref. 22, We compensate for the defect charge by applying a uniform background charge. Meta dynamics ${ }^{8}$ simulations were carried out in the NVT ensemble at $600 \mathrm{~K}$ ensured by a Nose-Hoover thermostat $\frac{2324}{20}$ for the ions, and using the PIVoT collective variable projected onto the (001) lattice direction. We choose $d_{0}=1.5 \AA$ and $\tau=0.4 \AA$ to ensure reasonable derivatives.

To understand the behavior of the PIVoT coordinate in detail, we compare the resulting vacancy trajectory

\begin{tabular}{|c|c|c|c|c|c|}
\hline $\begin{array}{c}\text { Barrier } \\
\text { static }\end{array}$ & $2 \times 2 \times 3$ & $\begin{array}{l}\text { DFTB } \\
3 \times 3 \times 6\end{array}$ & $4 \times 4 \times 6$ & $\begin{array}{l}\mathrm{DFT}^{12} \\
5 \times 4 \times 2\end{array}$ & $\begin{array}{l}\text { MSINDC } \\
2 \times 3 \times 2(\text { slab) }\end{array}$ \\
\hline $\mathrm{A}$ & 1.89 & 1.94 & 1.96 & 1.77 & - \\
\hline B & 1.41 & 1.03 & 0.88 & 0.69 & - \\
\hline $\mathrm{C}$ & 1.22 & 1.36 & 1.31 & 1.10 & - \\
\hline \multicolumn{6}{|l|}{ free } \\
\hline A & - & - & - & - & $\sim 0.96$ \\
\hline $\mathrm{C}$ & 1.44 & 1.09 & - & - & $\sim 1.22$ \\
\hline
\end{tabular}
to the center of gravity of the cavity in the $\mathrm{O}$ sublattice resulting from the vacancy. The void is determined by a procedure similar to cavity analysis in GeSbTe phase
Table I: $\mathrm{V}_{\mathrm{O}}^{2+}$ minimum energy diffusion barriers and the free energy diffusion barrier of the $\mathrm{C}$ process in $\mathrm{eV}$. Processes A,B occur within the equatorial plane of the corresponding Ti octahedron, process $\mathrm{C}$ is out-of plane. Nomenclature follows ref. 12, Values for MSINDO free energy barriers are averaged between forward and backward directions, since the (110) surface in these simulations breaks translational invariance.

change materials ${ }^{16}$ but explicitly without analyzing the void size. We examine volume elements of $0.1 \AA$ edge length, marking them as empty if no $\mathrm{O}$ atom is closer than $1.1 \AA$, just over $\frac{1}{2}$ of the $\mathrm{O}-\mathrm{O}$ neighbor distance in rutile. During a hopping event, empty voxels are filled by the moving $\mathrm{O}$ atom, while new empty elements appear behind its original position. The center of gravity of the empty elements transits continuously, with some thermal fluctuation. While the transition of the vacancy can be detected this way, its time scale is underestimated by a factor of 5, as shown by he comparison to the motion of the hopping $\mathrm{O}$ atom. In contrast, PIVoT reproduces the transition time very well. (See Supplemental Material at [URL will be inserted by publisher] for a detailed comparison.)

To verify the suitability of SCC-DFTB for the simulation of oxygen vacancy diffusion in $\mathrm{TiO}_{2}$, we calculate the minimum energy paths for the three symmetryinequivalent transitions a $\mathrm{V}_{\mathrm{O}}^{2+}$ can undergo using the nudged elastic band method for the $2 \times 2 \times 3$ and $3 \times 3 \times 6$ cells and adiabatic mapping for a $4 \times 4 \times 6$ supercell. The resulting diffusion barriers from the DFTB calculations turn out between 0.1 and $0.2 \mathrm{eV}$ higher than the GGA results, as shown Table [1, so the agreement is reasonable. Another important result in Table $\mathrm{I}$ is the strong supercell size dependence of the " $\mathrm{B}$ " barrier, which is connected to the occurrence of chains of $\mathrm{Ti}$ atoms linked by $\mathrm{O}$ double bridges along the (001) direction in rutile ${ }^{14}$. Careful analysis of the geometry shows that the supercell chosen by Iddir et.al. for their DFT calculations is well thought out to reduce this cell size effect. The slight increase of the $\mathrm{A}$ and $\mathrm{C}$ barriers with supercell size are attributable to stabilization of the vacancy equilibrium configuration in larger cells. Recent claims of strong charge state dependence of the $\mathrm{V}_{\mathrm{O}}$ diffusion barriers $\$ 13$ are dubious since the supercell size dependence is ignored there and $k$-point sampling is inadequate.

Even though barriers from the $2 \times 2 \times 3$ supercell are un- 


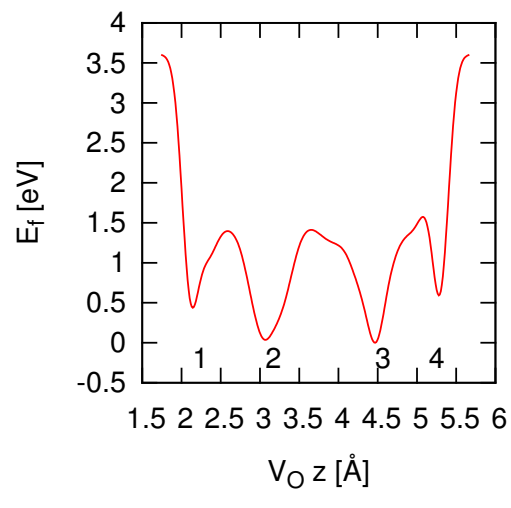

(a) $2 \times 2 \times 3$ FEP

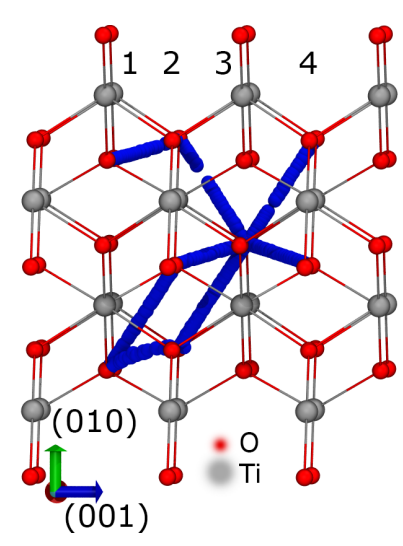

(b) $2 \times 2 \times 3$ trajectory

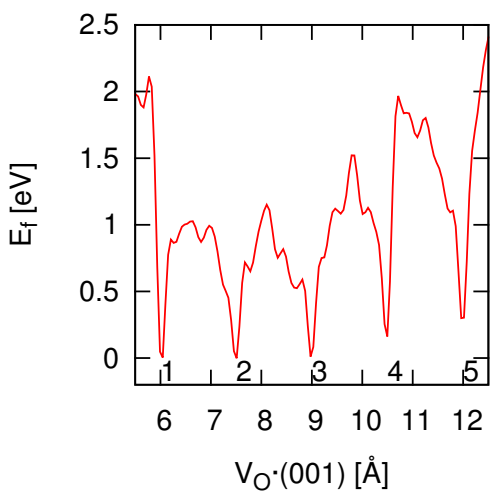

(c) Cutout of 3x3x6 FEP

Figure 1: (color online) (a) 130,000 step free energy profile of $\mathrm{V}_{\mathrm{O}}^{2+}$ along (001) in 2x2x3 supercell. Wall potentials constrain the motion of the vacancy at 1.7 and $5.8 \AA$. (b) Vacancy position shown every 100 fs as (dark) blue spheres, view approximately along the (100) axis, $\mathrm{Ti}$ and $\mathrm{O}$ atoms of the minimum energy structure of the $\mathrm{TiO}_{2}$ cell are shown for orientation. (c) cutout of the the FEP in the $3 \times 3 \times 6$ supercell after 75000 steps.

physically distorted, it is edifying to compare a free energy profile obtained from this cell to the minimum energy path (MEP). As Fig. 1b shows, the free energy profile (FEP) in Fig. 1a is averaged over several C-jumps between adjacent $\mathrm{TiO}$ planes, the profiles between minima 1-2 and 2-3 are each a thermodynamic mean of 3 distinct C-type transitions, while the $3-4$ barrier is only sampled along 2 routes. Artificial wall potentials at $\sim 1.7$ and $\sim 5.8 \AA$ were employed to keep the vacancy from approaching the periodic boundary. Therefore only the part between $2.5 \AA$ and $4.5 \AA$ can be analyzed. While an A transition would be possible, it was not observed. B Barriers will not be visible in the FEP, as the jumps are perpendicular to the analyzed direction. The mean value of the barriers weighted by the number of contributing transitions yields an activation free energy of $1.44 \pm 0.7 \mathrm{eV}$. This value is about $0.2 \mathrm{eV}$ higher than the MEP barrier, which is reasonable regarding the achievable accuracy and small cell size.

Fig. 1c shows a cutout of the FEP obtained after 75,000 meta dynamics iterations in the much larger $3 \times 3 \times 6$ supercell. In retrospect it was found that the artificial walls for the CV were placed too close to the periodic boundaries, so that atom jumps across the boundary occurred, leading to heavy artifacts on the simulation beyond 75,000 steps. A reasonably converged section of the FEP was selected by finding an area where minima of equal free energy coincide with $\mathrm{O}$ layers in the crystal. In this case, only two barriers could be analyzed marked 1-2 and 23 in Fig. 1c yielding a mean barrier of $1.09 \pm 0.13 \mathrm{eV}$. This barrier is somewhat smaller than in the MEP (cf. Tab. ID), which emphasizes the necessity for free energy calculation even of this seemingly simple process. As in the $2 \times 2 \times 3$ cell, all observed transitions are $\mathrm{C}$ type transitions, reinforcing the observation that long range migra-

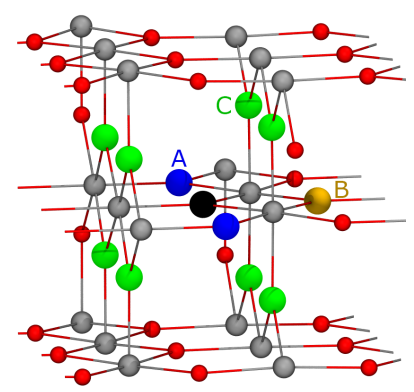

Figure 2: (color on line) Possible transitions for a $\mathrm{V}_{\mathrm{O}}$ defect in rutile. Initial vacancy position is marked in black.

tion of oxygen vacancies appears to be governed by this process.

For understanding the high preference for $\mathrm{C}$ jumps, it is necessary to examine the possible transitions closer, this time centered on the vacancy rather than a $\mathrm{Ti}$ atom. Fig. 2 shows all end points of the three types of nearest neighbor jumps a vacancy can undergo. The number of realizations for each event type varies strongly, with the ratio of A:B:C being 2:1:8. The topology shows that $\mathrm{B}$ jumps alone cannot lead to migration of the vacancy and A events can only lead to strictly linear migration along the (001) axis. In contrast, combinations of $\mathrm{C}$ jumps allow the vacancy to move in any direction. A combination a $\mathrm{B}$ and $\mathrm{C}$ jumps can lead to accelerated movement along

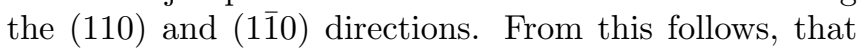
the rate-limiting step for vacancy diffusion in rutile is the C-type jump, which is the only hopping event during which the vacancy does not stay within the equatorial plane of any of its Ti neighbors.

In the current formulation, the PIVoT coordinate is 
limited to a single vacancy, but extension to a multivacancy capable M-PIVoT formulation for a known number of vacancies can be achieved in a straight-forward manner by clustering the strongly weighted reference positions, e.g. using the $k$-means algorithm 25 . Overcoming the current limitation to a static, externally provided reference lattice is equally straight forward, at least in the case of compounds. Without loss of generality, regarding an $\mathrm{AB}$ compound, the expected $\mathrm{B}$ positions can be inferred from the A sublattice. E.g., rutile is composed of isosceles triangles with $\mathrm{Ti}$ vertices and edges of $\sim 3.0$ and $2 \mathrm{x} \sim 3.6 \AA$ in length. Reference positions of sufficient quality can be calculated by taking the center of gravity of every group of three $\mathrm{Ti}$ atoms no further apart than $3.6 \AA$ plus a commensurate allowance for thermal fluctuation. Similar rules can be formulated for any compound where the species of interest is at least 3-fold coordinated.

Our simulation results demonstrate that meta dynamics are a useful tool explore and understand diffusion mechanisms in complex materials. In contrast to minimum energy path search approaches, where the start and end configurations must be provided by an educated guess of the researcher, and thus every possible process must be foreseen, no a-priori assumptions on the diffusion mechanism are imposed. The dangers of this reliance on chemical intuition have been demonstrated, e.g. in the case of surface adatom diffusion 26 . A collective variable based (meta) dynamics approach avoids this pitfall that is even deeper for vacancies, which are an emergent property of the distribution if indistinguishable atoms.

Besides serving as a collective variable for free energy sampling, the PIVoT coordinate also shows great potential for use as an event detection method in parallel replica dynamics 11 and related approaches. In contrast to the currently favored approach of using differences in atom positions after steepest descent relaxation of MD snapshots, the PIVoT coordinate is computationally so efficient that it can be evaluated on the fly and events can be detected immediately, reducing the computational cost while increasing the accuracy of event time measurement. Beyond tracking a vacancy outright, it is trivial to use the PIVoT coordinate as part of any other vector based variable to describe a system, e.g. distances, angles, coordination numbers etc. The vacancy tracking is independent of the vacancy charge state and can be applied under any conditions as long as the interatomic potential remains valid. Combined with Born-Oppenheimer dynamics, as shown here, the charge distribution is always determined by the ionic arrangement. This allows to analyze correlations between ion dynamics and electronic rearrangements.
Applied to the behavior of $\mathrm{V}_{\mathrm{O}}^{2+}$ defects in rutile, we demonstrated that great care must be taken in the choice of models and computational details. Furthermore, our free energy surface samplings show that not the lowest barrier pathway dominates the $\mathrm{V}_{\mathrm{O}}^{2+}$ diffusion, but the $\mathrm{C}$ hop, which possesses the largest number of realizations per $\mathrm{O}$ site and provides the largest connectivity between lattice sites. While the good agreement between MEP and free energy paths in thermal equilibrium is not very surprising in a crystalline solid, our results demonstrate that free energy path sampling is in deed feasible in the complex system of reduced titania. This opens the door to answering much more challenging questions regarding the effect of external driving forces such as electric fields or temperature gradients and their relative impact on the memristive effect in different materials, which is crucial for understanding and designing memristor devices 27 .

The PIVoT coordinate was shown to be well suited to drive meta dynamics simulation of oxygen vacancy diffusion in titania and to analyze the resulting trajectories. We demonstrate that in combination with potentials that offer a good compromise between efficiency, accuracy and transferability such as SCC-DFTB, this vacancy coordinate allows examining the free energy landscape of vacancy diffusion safe from the pitfalls of previously existing mappings between atomic and vacancy motion. We show that the diffusion of $\mathrm{V}_{\mathrm{O}}^{2+}$ defects is dominated by out-of-plane hopping events even though lower barrier jumps may occur.

The methods outlined in this work are applicable far beyond titania. The PIVoT coordinate can be used to analyze and drive vacancy dynamics in any solid, as long as the atomic arrangement does not undergo any severe modification. This allows to tackle questions of vacancy dynamics under external driving forces, such as electric fields or temperature gradients.

The authors thank Dr. Peter Deák of the BCCMS for discussions on the simulation of $\mathrm{V}_{\mathrm{O}}$ in $\mathrm{TiO}_{2}$. We are grateful to Mrs. Svea große Holthaus at the BCCMS and Professor Stephan Irle at Nagoya University for advice about meta dynamics simulation. We thank Dr. Bálint Aradi at the BCCMS for his help in coupling the PLUMED code to DFTB ${ }^{+}$. We thank Prof. Stephen Elliott and Dr. Taehoon Lee of the University of Cambridge for advice on void detection analysis. All simulations were carried out using the $\mathrm{DFTB}^{+} \operatorname{code}{ }^{28}$, meta dynamics simulation was performed by an extended version of the PLUMED code ${ }^{29}$ version 1.3. Model representations were prepared using VMD ${ }^{30}$. J.M.K is grateful for funding from the DFG.

\footnotetext{
* jan.knaup@bccms.uni-bremen.de

$\dagger$ frauenheim@bccms.uni-bremen.de

1 D.-H. Kwon, K. M. Kim, J. H. Jang, J. M. Jeon, M. H. Lee, G. H. Kim, X.-S. Li, G.-S. Park, B. Lee, S. Han, et al.,
}

nature nanotechnology 5, 148 (2010).

S.-G. Park, B. Magyari-Köpe, and Y. Nishi, IEEE Electron

Device Letters 32, 197 (2011).

D. B. Strukov, G. S. Snider, D. R. Stewart, and R. S. 
Williams, Nature 453, 80 (2008), URL http://dx.doi. org/10.1038/nature06932

J. P. Strachan, J. J. Yang, R. Münstermann, A. Scholl, G. Medeiros-Ribeiro, D. R. Stewart, and R. S. Williams, Nanotechnology 20, 485701 (2009), URL http://stacks . iop.org/0957-4484/20/i=48/a=485701.

${ }^{2}$ R. Waser, R. Dittmann, G. Staikov, and K. Szot, Adv. Mater. 21, 2632 (2009), URL http://dx.doi.org/10. 1002/adma. 200900375

${ }^{3}$ M. D. Pickett, D. B. Strukov, J. L. Borghetti, J. J. Yang, G. S. Snider, D. R. Stewart, and R. S. Williams, J. Appl. Phys. 106, 074508 (pages 6) (2009), URL http://link. aip.org/link/?JAP/106/074508/1.

${ }^{4}$ L. A. Bursill and B. G. Hyde, Prog. Sol. Stat. Chem. 7, 177 (1972).

M. Marezio, D. B. McWhan, P. D. Dernier, and J. P. Remeika, J. Solid State Chem. 6, 213 (1973), ISSN 0022-4596, URL http://www.sciencedirect.com/ science/article/pii/0022459673901849

L. Liborio and N. Harrison, Phys. Rev. B 77, 104104 (2008).

L. Liborio, G. Mallia, and N. Harrison, Phys. Rev. B 79, 245133 (2009).

${ }^{5}$ C. T. Campbell and C. H. F. Peden, $\quad$ Science $\quad 309, \quad 713 \quad$ (2005), http://www.sciencemag.org/content/309/5735/713.full.pdf, URL http://www.sciencemag.org/content/309/5735/ 713.short

${ }^{6}$ O. Bikondoa, C. L. Pang, R. Ithnin, C. A. Muryn, H. Onishi, and G. Thornton, Nat Mater 5, 189 (2006/), URL http://dx.doi.org/10.1038/nmat1592.

P. L. Gai-Boyes, Catalysis Reviews 34, 1 (1992), http://www.tandfonline.com/doi/pdf/10.1080/01614949208 URL http://www.tandfonline.com/doi/abs/10.1080/ 01614949208021918

R. Wanbayor, P. Deák, T. Frauenheim, and V. Ruangpornvisuti, Computational Materials Science 58, 24 (2012), ISSN 0927-0256, URL http://www.sciencedirect.com/ science/article/pii/S092702561200033X.

R. Wanbayor, P. Deák, T. Frauenheim, and V. Ruangpornvisuti, The Journal of Chemical Physics 134, 104701 (pages 6) (2011), URL http://link .aip.org/link/?JCP/ 134/104701/1.

P. Scheiber, M. Fidler, O. Dulub, M. Schmid, U. Diebold, W. Hou, U. Aschauer, and A. Selloni, Phys. Rev. Lett. 109, 136103 (2012), URL http://link.aps.org/doi/10. 1103/PhysRevLett.109.136103.

W.-P. Dow, Y.-P. Wang, and T.-J. Huang, Journal of Catalysis 160, 155 (1996), ISSN 0021-9517, URL http://www . sciencedirect.com/science/article/ pii/S0021951796901359

A. Laio and M. Parrinello, Proceedings of the National Academy of Sciences 99, 12562 (2002), http://www.pnas.org/content/99/20/12562.full.pdf+html, URL http://www.pnas.org/content/99/20/12562. abstract

${ }^{9}$ G. Gobbo, A. Laio, A. Maleki, and S. Baroni, Phys. Rev. Lett. 109, 150601 (2012), URL http://link.aps.org/ doi/10.1103/PhysRevLett.109.150601.

${ }^{10}$ G. Díaz Leines and B. Ensing, Phys. Rev. Lett. 109, 020601 (2012), URL http://link.aps.org/doi/10.1103/

\section{PhysRevLett.109.020601}

A. F. Voter, Phys. Rev. B 57, R13985 (1998), URL http: //link.aps.org/doi/10.1103/PhysRevB.57.R13985.

${ }^{12}$ H. Iddir, S. Öüut, P. Zapol, and N. D. Browning, Phys. Rev. B 75, 073203 (2007).

13 A. M. Asaduzzaman and P. Krüger, J. Phys. Chem. C 114, 19649 (2010).

14 J. M. Knaup, M. Wehlau, and T. Frauenheim, in $M R S$ Proceedings (2012), vol. 1430, pp. 1430mrss12-1430-e0810.

${ }^{15}$ K. Jug, N. N. Nair, and T. Bredow, Phys. Chem. Chem. Phys. 7, 2616 (2005), URL http://dx.doi.org/10.1039/ B502507A

16 J. Akola and R. Jones, phys. rev. B 76, 235201 (2007).

17 T. H. Lee and S. R. Elliott, Phys. Rev. B 84, 094124 (2011), URL http://link .aps .org/doi/10.1103/ PhysRevB.84.094124

18 Note1, for the sake of simplicity and without loss of generality we assume for a moment that the transition barrier is symmetrical between the adjacent minima.

19 T. Frauenheim, G. Seifert, M. Elstner, Z. Hajnal, G. Jungnickel, D. Porezag, S. Suhai, and R. Scholz, phys. stat. sol. (b) 217, 41 (2000).

B. Aradi, B. Hourahine, and T. Frauenheim, J. Phys. Chem. A 111, 5678 (2007).

20 G. Dolgonos, B. Aradi, N. H. Moreira, and T. Frauenheim, Journal of Chemical Theory and Computation 6, 266 (2010), http://pubs.acs.org/doi/pdf/10.1021/ct900422c, URL http://pubs.acs.org/doi/abs/10.1021/ ct900422C

${ }^{21}$ H. J. Monkhorst and J. D. Pack, Phys. Rev. B 13, 5188 (1976), URL http://link.aps.org/doi/10.1103/

2191 BhysRevB.13.5188

${ }^{22}$ P. Deák, B. Aradi, and T. Frauenheim, Phys. Rev. B 86, 195206 (2012), URL http://link .aps.org/doi/10.1103/ PhysRevB.86.195206

S. Nosé, J. Chem. Phys. 81, 511 (1984), URL http:// link. aip.org/link/?JCP/81/511/1

${ }^{24}$ W. G. Hoover, Phys. Rev. A 31, 1695 (1985), URL http: //link.aps.org/doi/10.1103/PhysRevA.31.1695

${ }^{25}$ J. A. Hartigan and M. A. Wong, Journal of the Royal Statistical Society. Series C (Applied Statistics) 28, pp. 100 (1979), ISSN 00359254, URL http://www . jstor.org/ stable/2346830

26 C. Liu, J. Cohen, J. Adams, and A. Voter, Surface Science 253, 334 (1991), ISSN 0039-6028, URL http://www. sciencedirect.com/science/article/ pii/003960289190604Q.

J. J. Yang, D. B. Strukov, and D. R. Stewart, Nat Nano 8, 13 (2013), URL http://dx.doi.org/10.1038/nnano. 2012.240

28 DFTB $^{+}$, URL http://www.dftb-plus.info/

29 M. Bonomi, D. Branduardi, G. Bussi, C. Camilloni, D. Provasi, P. Raiteri, D. Donadio, F. Marinelli, F. Pietrucci, R. A. Broglia, et al., Computer Physics Communications 180, 1961 (2009), ISSN 0010-4655, URL http://www.sciencedirect.com/science/article/ pii/S001046550900157X.

${ }^{0}$ W. Humphrey, A. Dalke, and K. Schulten, Journal of Molecular Graphics 14, 33 (1996), URL http://www.ks. uiuc.edu/Research/vmd/ 